\title{
Pulmonary Artery Intimal Sarcoma Diagnosed as Pulmonary Embolism
}

\author{
Ali Abbood, Hareer Al Salihi, Olatunji Otegbeye, Mario Madruga, and S. J. Carlan,
}

\section{ABSTRACT}

Background: Pulmonary artery intimal sarcoma (PAIS) is a rare malignancy of vasculature that carries a very poor prognosis that is often misdiagnosed as a pulmonary embolism due to overlapping clinical and radiological features. It is a very aggressive malignancy with surgery the mainstay of initial management. Chemotherapy is normally given postoperatively, although it is not clear if chemo and radiotherapy bring any improvement, some data report an increase in survival in patients who received multimodality therapy vs single therapy.

Case: A 48 year-old female was initially diagnosed with a pulmonary embolism based on clinical presentation and imaging. She was treated vigorously but continued to show no improvement after two weeks. She underwent an open thrombectomy and a large concurrent pulmonary artery intimal sarcoma (PAIS) was found. The mass was adherent to the intima and extended into the left and right pulmonary arteries. The tumor was not fully resected and tissue results showed poorly differentiated sarcoma. A treatment plan was then initiated to include systemic chemotherapy. She developed metastatic disease despite receiving multimodal therapy and died with within 2 years from the initial diagnosis.

Conclusion: Persistent symptoms in patients diagnosed and treated adequately for pulmonary artery thrombosis should indicate a possible comorbid condition including sarcoma. This is particularly the case in the older age group with imaging that suggests central embolism despite treatment.

Keywords: Pulmonary artery intimal sarcoma; Pulmonary embolism; Sarcoma.

Published Online: July 24, 2021

ISSN: $2736-5476$

DOI: $10.24018 /$ ejclinicmed.2021.2.3.85
A. Abood*
Orlando Regional Healthcare, Department of Internal Medicine,
Orlando, Florida, USA.
(e-mail:
Ali.Abbood@orlandohealth.com)
H. A. Salihi
Orlando Regional Healthcare,
Department of Internal Medicine,
Orlando, Florida, USA
(e-mail:
Hareer.AlSalihi@orlandohealth.com)
O. Otegbeye
Orlando Regional Healthcare,
Department of Internal Medicine,
Orlando, Florida, USA.
(e-mail:
Olatunji.Otegbeye@ orlandohealth.com)
M. Madruga
Orlando Regional Healthcare,
Department of Internal Medicine,
Orlando, Florida, USA.
(e-mail:
Mario.madruga@orlandohealth.com)
S. J. Carlan*
Orlando Regional Healthcare, Division
of Academic Affairs and Research,
Orlando, Florida, USA.
(e-mail: stevecarlan@gmail.com)
*Corresponding Author

\section{INTRODUCTION}

Pulmonary artery intimal sarcoma (PAIS) is a rare endovascular malignancy with poor prognosis. In addition to the rarity of this disease, the symptoms are non-specific, and it is commonly misdiagnosed as pulmonary embolism, making early diagnosis challenging. PAS is an aggressive tumor with a poor reported prognosis in published series being 1.5 months without any kind of treatment [1]. Owing to the limited number of cases there are very few small case series describing the management of this tumor which we aim to discuss in this case report.

\section{CASE REPORT}

A 48 year old female with no past medical history, who presented with shortness of breath and right apical chest pain for the past 2 weeks. One month prior to her initial presentation, she was diagnosed with saddle pulmonary embolism at an outside facility and had received tissue plasminogen activator (tPA) and had penumbra aspiration thrombectomy of right pulmonary trunk, right pulmonary artery, some segmental branches and left pulmonary artery. However, the patient was still having persistent shortness of breath which required multiple admissions. CT scan of the chest showed extensive central pulmonary embolism almost entirely filling the central pulmonary arteries with mild extension into upper as well as lower lobar arteries

An echocardiogram showed a dilated right ventricle, mild MR (mitral regurgitation), mild TR (tricuspid regurgitation) and left ventricular ejection fraction of $60 \%$. The patient underwent an open thrombectomy, which showed a mass that appeared to be tumor in the pulmonary artery rather than thrombus. The mass was adherent to the intima and extended 
into the left and right pulmonary arteries. It was carefully dissected however it was adherent in many areas and was not resected fully. Biopsy was sent for Pathology and results showed poorly differentiated sarcoma (Fig. 2), with atypical spindle cells and negative staining for the following stains: desmin, myogenin, and keratin.

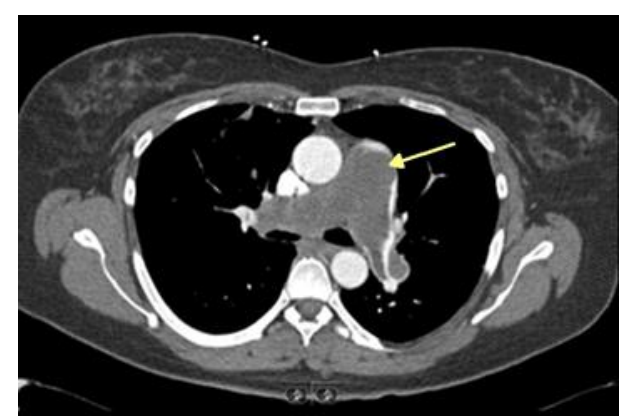

Fig. 1. Extensive pulmonary embolism (yellow arrow).

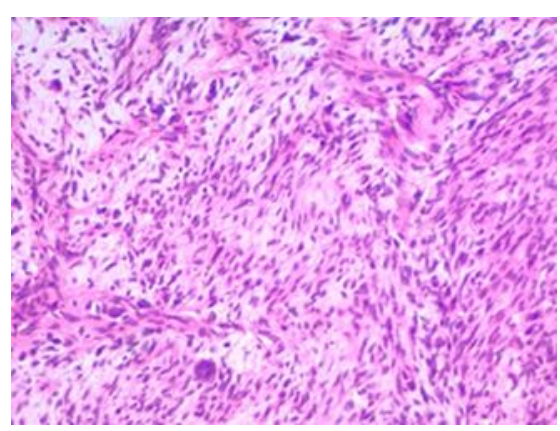

Fig. $240 x$ H \& E stain. Tissue section shows the tumor cells which are arranged in fascicles with myxoid areas are composed of pleomorphic atypical spindle cells with mi-totic activity and necrosis.

She was evaluated by a multidisciplinary team including, oncologist, radiologist, pathologist, cardiovascular surgeons, and pulmonologist. A treatment plan was then initiated to include systemic chemotherapy with doxorubicin, ifosfamide and mesna (AIM therapy) for total of 8 cy-cles. The patient was started on nivolumab and imatinib for c-KIT (tyrosineprotein kinase Kit) amplification of the tumor. She continued to follow outpatient with CT chest/abdomen and pelvis every 2 months. One year after her diagnosis and surgery, patient had CT that showed hypo-dense fullness of the truncated right upper lobe pulmonary artery and thickening of the main pulmonary artery valve that were concerning for recurrent tumor. She had right upper lobectomy and reconstruction of main pulmonary artery with cardiothoracic surgery and was recovering well. However, 2 months after the surgery another CT chest/ abdomen showed tho-racic lymphadenopathy with new pancreatic head mass. Laparoscopic biopsy showed high grade sarcoma. She was then transitioned to comfort care with hospice and passed away less than two years after her initial diagnosis was made.

\section{DISCUSSION}

PAIS is a an extremely rare sarcoma. It originates from intimal cells of the pulmonary artery, and clinically it can be difficult to diagnose and distinguish it from pulmonary embolisms [2]. Some of the most prevalent complaints when the patient first presents include progressive dyspnea, chest pain, weight loss and cough. For example, a pulmonary embolism was expected in this present case. The patient was previously diagnosed with saddle pulmonary embolism at an outside facility and had received tPA and had penumbra aspiration thrombectomy of right pulmonary trunk, right pulmonary artery, some segmental branches and left pulmonary artery. PAIS was suspected until the patient was seen at our hospital and the patient went for an open thrombectomy.

In a study of PAIS vs Pulmonary Artery Thromboembolism (PTE), almost one half $(42 \%)$ of PAIS patients had a diagnosis that was delayed for a mean day of 268 days. As expected, the most common incorrect diagnosis was a pulmonary embolism in 54\%. [3] It points out that because of it being a rare disease many radiologists are not aware of the findings that help differentiate the two disorders from one another. In CT findings, no PAIS case had a tubularpolypoid shape, while they found that two-thirds of the PTEs were found to have tubular -polypoid shape which probably represents movement of the thrombus from DVT. Half of the PTE's were shown to have DVTs compared to none of the PAIS [3]. PAIS had a higher attenuation and were significantly more heterogeneous than did PTE. Furthermore, the wall eclipse sign was a significant finding, which is seen in PAIS but not in PTE. When PAIS is suspected an MRI can be very useful in the differential, because PAIS showed diffusion restriction and heterogeneous enhancement that were not seen in PTE [3]. It was also noted that clinically PAIS was more common in young-er patients. Also, there were lower levels of D-dimer and BNP when comparing PAIS to PTE.

Surgical treatment remains the mainstay of management for PAIS. Once our patient, went for an open thrombectomy and the surgeons realized it was more of a mass than a thrombus and was unable to fully resect the mass because of its adherence to the intima. In one case report, a patient who had a full radical resection of the PAIS had been alive and symptom free for 16 months post-surgery [4]. Typically, chemotherapy is offered postoperatively, although it is not clear if radiotherapy and chemotherapy bring any measurable improvement. Recent data re-ported an improvement in survival in cases who received multimodality therapy versus single therapy. Multiple agents have been reported in the literature as possibly useful including an-thracyclines, ifosfamide, gemcitabine, taxanes, platinums, and immunotherapy. Anthracyclines either alone or in combination are the most commonly used agents [5]. Since our patient was unable to have full resection, the multidisciplinary team decided to start the patient on postoperative treatment of doxorubicin, ifosfamide, and mensa.

\section{CONCLUSION}

Persistent symptoms in patients diagnosed and treated adequately for pulmonary artery thrombosis should indicate a possible comorbid condition including sarcoma. This is particularly the case in the older age group with imaging that suggests central embolism despite treatment.

When you submit your final version, after your paper has been accepted, prepare it in two-column format, including figures and tables. 


\section{REFERENCES}

[1] Krüger I, Borowski A, Horst M, de Vivie ER, Theissen P, GrossFengels W. Symptoms, diagno-sis, and therapy of primary sarcomas of the pulmonary artery. Thorac Cardiovasc Surg. 1990;38:91-5.

[2] Aso T, Terao M, Endo H, Tabata M. A case of pulmonary artery intimal sarcoma successfully treated using concurrent chemoradiotherapy and subsequent chemotherapy.Int Cancer Conf J. 2019 Jan 25;8(2):71-76. doi: 10.1007s13691-018-00356-0. eCollection 2019 Apr.

[3] Kim C, Kim MY, Kang JW, Song JS, Lee KY, Kim SS.Pulmonary Artery Intimal Sarcoma versus Pulmonary Artery Thromboembolism: CT and Clinical Findings. Korean J Radiol. 2018 Jul-Aug;19(4):792802. doi: 10.3348/kjr.2018.19.4.792. Epub 2018 Jun 14.

[4] Yamamoto Y, Shintani Y, Funaki S, Aggressive Surgical Resection of Pulmonary Artery Intimal Sarcoma. Ann Thorac Surg. 2018 Oct;106(4):e197-e199. doi: 10.1016/j.athoracsur.2018.03.072. Epub 2018 May 3.

[5] Chiola I, Belgioia L, Vaccara EML, Gusinu M, Corvò R. Reirradiation of pulmonary artery intimal sarcoma: A case report. Clin Case Rep. 2019 May 29;7(7):1342-1346. doi: 10.1002/ccr3.2216. eCollection 2019 Jul. 